\title{
OBSERVING BEAM MOTION USING INFRARED INTERFEROMETRY *
}

\author{
J. M. Byrd ${ }^{\dagger}$, M. Martin, W. McKinney \\ Lawrence Berkeley National Laboratory, One Cyclotron Road, Berkeley, California 94720
}

\begin{abstract}
Phase noise in the RF master oscillator driving synchrotron oscillations of the beam has been identified as one of the dominant sources of noise in the infrared beamline at the Advanced Light Source. We present measurements of the effect of the electron beam motion in a Fourier transform interferometer (FTIR) detector. This form of detector may be sensitive to very small beam motions.
\end{abstract}

\section{INTRODUCTION}

Interest in infrared (IR) synchrotron radiation has grown dramatically in the past few years. However, measurements at the synchrotron IR source have proven to be very sensitive to both beam motion and mechanical motion of the endstation $[1,2,3]$. The beam motion can result from a combination of self-excited and driven motion, ranging in frequency from a few hertz to hundreds of kilohertz. IR beamline 1.4.3 at the Advanced Light Source (ALS) located at Lawrence Berkeley National Laboratory is no exception to these problems.

Over the past year, we have made an effort to identify and resolve noise issues in the beamline as well as understand the mechanisms for observing the noise. In particular, we have made an detailed study of beam motion excited by phase noise in the master oscillator (MO) of the RF system since this was determined to be one of the dominant noise sources. This has led us to the conclusion that the interferometer used in the endstation may serve as an excellent general diagnostic for observing very small beam motion. This paper summarizes our measurements to date and provides several hypotheses for the sensitivity to beam motion. Section 2 provides a description of the IR source, beamline, and interferometer. Section 3 presents measurements of the beam motion excited by MO phase noise.

\section{MEASUREMENT SETUP}

The ALS is a $1.5-1.9 \mathrm{GeV}$ electron storage ring optimized for producing high brightness synchrotron radiation. The synchrotron light is collected from the 1.4 bending magnet with source parameters listed in Table 1.

The light passes through a $10 \mathrm{mrad}$ vertical and $40 \mathrm{mrad}$ horizontal opening, as schematically drawn in Figure 1.

\footnotetext{
* This work was supported by the U.S. Dept. of Energy under Contract No. DE-AC03-76SF00098.

† JMByrd@lbl.gov
}

\begin{tabular}{cll} 
Parameter & Description & Value \\
\hline$E$ & Beam energy & $1.5-1.9 \mathrm{GeV}$ \\
$I$ & nominal current range & $100-400 \mathrm{~mA}$ \\
$\epsilon_{x}, y$ & x,y emittance@ 1.9 GeV & $6,0.06 \mathrm{~nm}-\mathrm{rad}$ \\
$\beta_{x}, y$ & x,y beta functions & $0.45,19.0 \mathrm{~m}$ \\
$\alpha_{x}, y$ & x,y alpha functions & $0.43,-7.7$ \\
$\eta_{x}$ & dispersion function & $4.18 \mathrm{~cm}$ \\
$\eta_{x}^{\prime}$ & dispersion slope & 0.13 \\
\hline
\end{tabular}

Table 1: ALS IR source parameters.

This light is deflected vertically by 0.5 meter, then is refocused outside the shield wall by an ellipsoidal mirror, $\mathrm{m} 2$. A "switchyard" then contains a series of optics to collimate the IR beam and distribute the beam to one of three end stations.

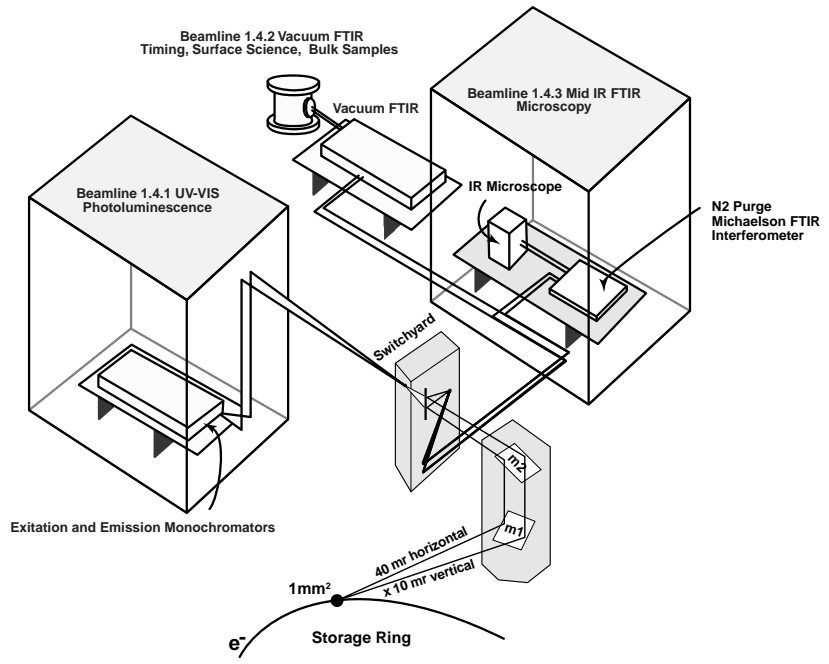

Figure 1: Schematic layout of the ALS infrared beamline.

BL 1.4.3 directs the collimated synchrotron light into a Nicolet Magna 760 FTIR bench. The modulated light is then passed through a Nic-Plan IR microscope that can perform both transmission and reflection measurements. BL 1.4.2 uses the synchrotron light for the input of a Bruker IFS 66v/S vacuum FTIR spectrometer. This IFS 66v/S instrument has a wide spectral range, $50 \mathrm{~cm}-1$ to $25,000 \mathrm{~cm}$ 1 , and it has step-scan capabilities in addition to rapid-scan to enable fast timing measurements. BL 1.4.1 uses the UV part of the spectrum from the 1.4 front end to do photoluminescence and related studies up to $6 \mathrm{eV}$. 


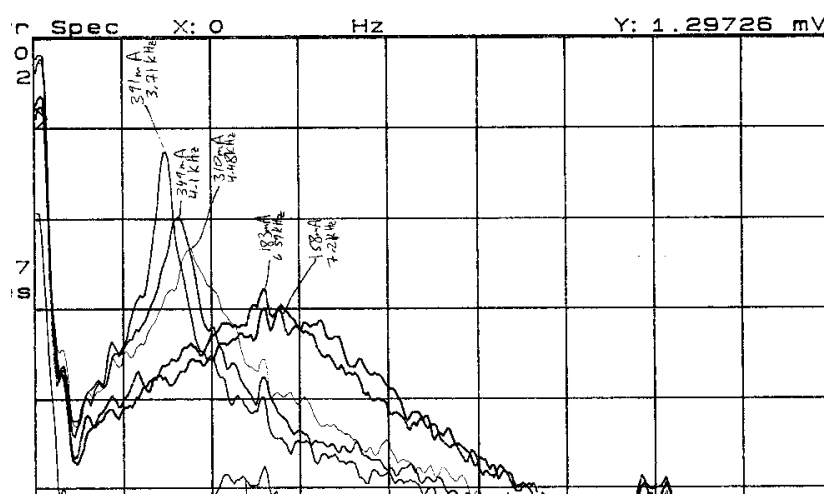

Figure 2: Noise measured in the FTIR as a function of current. The highest peak was recorded at a current of $391 \mathrm{~mA}$ and the lowest at $158 \mathrm{~mA}$. The frequency axis ranges from 0 to $23 \mathrm{kHz}$.

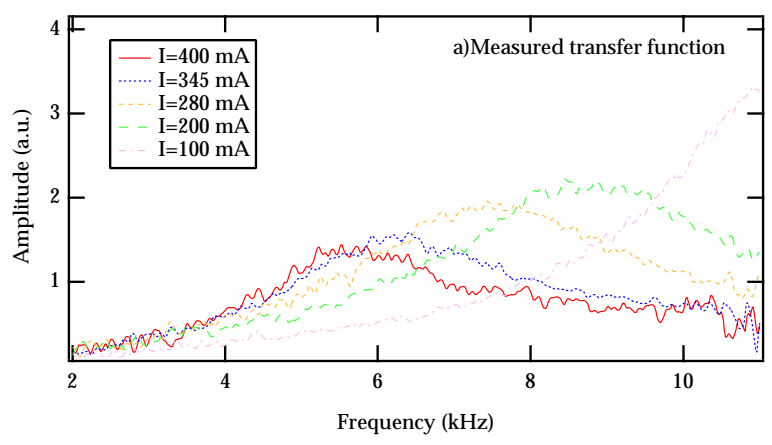

Figure 3: Transfer functions of the FTIR response from exciting the cavity phase at various beam currents.

\section{MEASUREMENTS}

To study the spectrum of noise in the FTIR, we fixed the moving mirror in the interferometer and recorded the output of the detector on a spectrum analyzer. An example of this measurement is shown in Fig. 2, which shows a noise peak at about $3.7 \mathrm{kHz}$ which decreases in frequency and increases in amplitude with increasing beam current.

We recognized this as possibly being a form of synchrotron oscillations known as the Robinson mode that could be driven by broadband noise in the RF system. By measuring the noise at various points in the RF system, we determined that the dominant source was phase noise in the $500 \mathrm{MHz}$ master oscillator. The details of beam motion driven by phase noise are discussed elsewhere[4].

In order to further demonstrate the effect of phase noise on the IR beamline, we deliberately excited the cavity by modulating the cavity phase with white noise and measured the response as a function of beam current as shown in Fig. 3a. Shown in Fig. 4a is a calculation of the expected response at the same beam currents, assuming that the motion results from energy oscillations of the beam at the source point.

To find the spectrum of beam motion driven by the MO

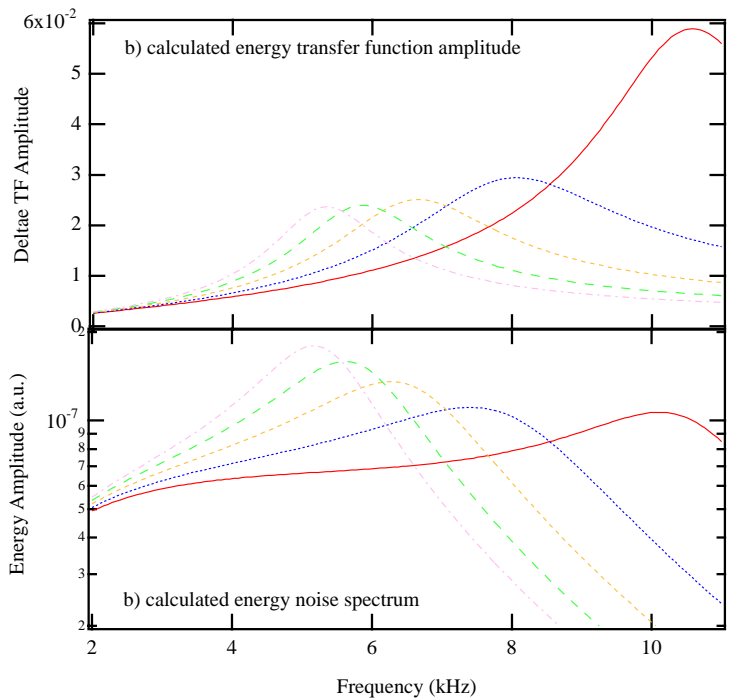

Figure 4: a) Calculated amplitude of the transfer function. This compares well with the measured values in Fig. 3. b) Calculated noise spectrum using the spectrum of MO phase noise and the calculated beam energy transfer function. This shows qualitative agreement with the measured noise in Fig. 2.

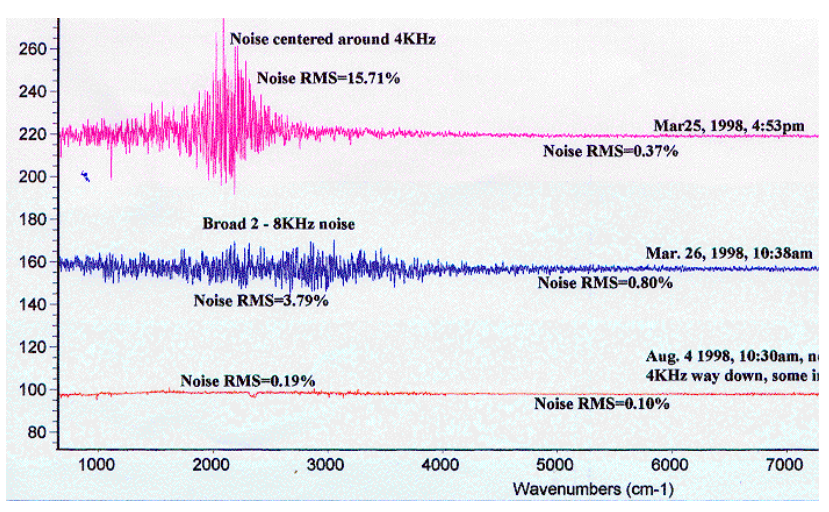

Figure 5: Three IR spectra showing the effects of the MO phase noise. The upper two traces were taken with the old MO, the bottom trace with the new MO.

phase noise, we computed the product of the MO phase noise determined from catalog values with the transfer function computed in Fig. 4a. This is shown in Fig. 4b. This has the same characteristic shape as the measured noise in Fig. 2. Replacement of the existing of the existing MO by a lower noise model reduced this motion by a factor of 5 .

The net result of the reduction in phase noise can be observed in Fig 5, which shows several spectral scans taken before and after the MO phase noise was reduced.

Although we succesfully identified the source of the noise and reduced it, we still did not understand how the beam motion created a signal in the interferometer. To further study this, we measured the beam motion driven by the 
noisy MO at very low beam current, where we could independently determine the amplitude of synchrotron oscillations and thus the amplitude of energy oscillations at the horizontally dispersive IR source point. Shown in Fig. 6 is a plot of the FTIR noise at low current for the old and new MO. We independently determined that the amplitude of beam energy oscillations was $2.8 \mathrm{e}-5$ (relative to the nominal energy), corresponding the horizontal position and angle oscillations of $1.6 \mu \mathrm{m}$ and $3.8 \mu \mathrm{rad}$, respectively.

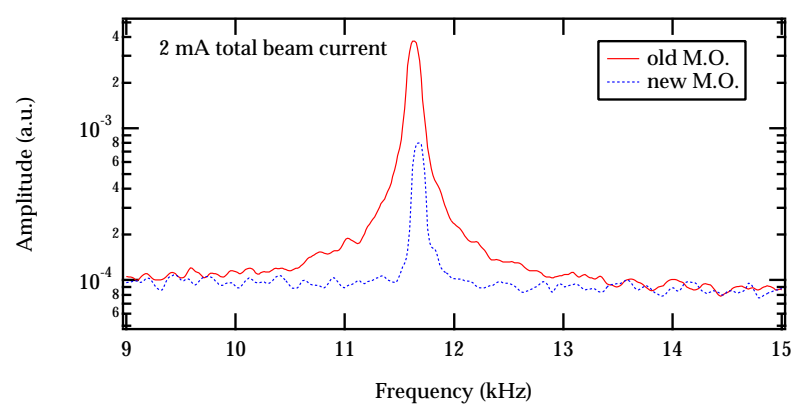

Figure 6: Motion driven by MO phase noise at low beam current for two MO sources. The reduction is equal to the reduction in the phase noise.

Given such small beam motion with a signal/noise of about 40 indicated that the interferometer was sensitive to beam motion of about $40 \mathrm{~nm}$ ! This suggests that the interferometer may be used as a beam motion detector with extremely high sensitivity. Following are two possible noise mechanisms which we are currently studying. Consider the schematic diagram of a Michelson interferometer as shown in Fig. 7. If the incoming light is not normal to the mirror (M2), there is a path length difference in between the two arms $L 1$ and $L 2$ of $\Delta L \theta^{2}$, where $\Delta L$ is the difference $L 2-L 1$ and $\theta$ is the incident light angle. Angular variations of the incident light can occur from position variations of the light incident of the ellipsoidal focussing mirror or deviations from optical flatness of any of the subsequent mirrors. A second mechanism is nonflatness of the beam splitter. In this case, transverse motion of the incident light beam will result in a path length difference in the two arms.

\section{CONCLUSIONS}

Phase noise in the RF master oscillator has been identified as one of the dominant sources of noise in the ALS infrared beamline. The problem has been substantially reduced by replacing the MO with a lower noise source. Interferometric measurements of synchrotron radiation are very sensitive to beam motion and may be used as a sensitive beam diagnostic. The means by which the noise is observed at the ALS is still under investigation. We would like to thank H. Zyngier for many useful discussions.

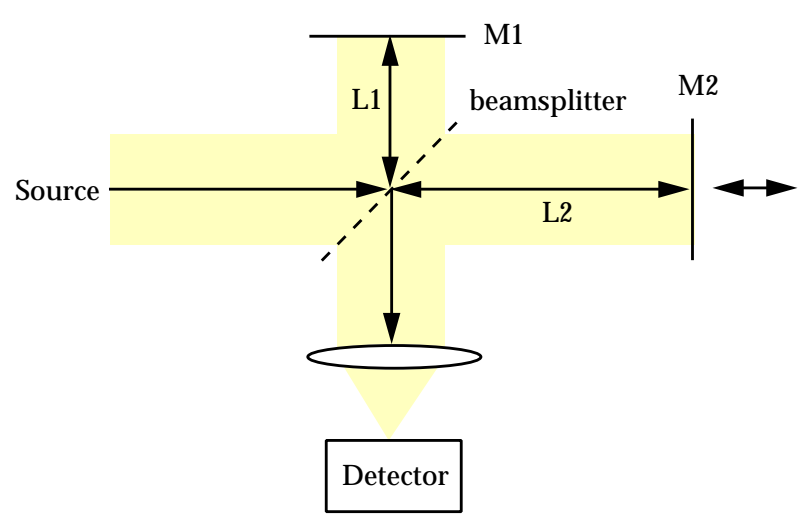

Figure 7: Schematic diagram of a Michelson interferemeter. Path length differences can arise from variations of the incident light angle or position variations combined with a nonflat beamsplitter.

\section{REFERENCES}

[1] A. R. Hight Walker, U. Arp, G. T. Fraser, T. B. Lucatorto, J. Wen, SPIE 3153, 42, 1997.

[2] R. Biscardi, G. Ramirez, G. P. Williams, C. Zimba, Rev. Sci. Inst. 66, 1856, 1995.

[3] G. Rakowsky, L. R. Highey, Rev. Sci. Inst. 66, 1856, 1995.

[4] J. M. Byrd, Effects of phase noise in heavily beam loaded storage rings, these proceedings. 